\title{
Velocity Fields in 9 Southern Planetary Nebulae
}

Krzysztof Gesicki

CA UMK, ul.Gagarina 11, Torun, Poland

Agnes Acker

Observatoire de Strasbourg, 11, rue de l'universite, Strasbourg, France

\begin{abstract}
In 1997 and 1998 we observed $16 \mathrm{PN}$ with the ESO $1.4 \mathrm{~m}$ CAT telescope, in the $\mathrm{H} \alpha$ and [NII] lines. We selected 9 PN with fairly symmetric line profiles, these PN we analyzed with the "Torun codes".
\end{abstract}

The derived expansion velocities are presented in Table 1 . Column $v_{\text {exp }}$ refers to the value of a constant velocity field, or in case of acceleration (i.e when $\Delta v>0$ ), to the mass weighted average velocity. The differences between velocities at the outer and inner nebular radius are given in next column: $\Delta v$. In the column $v_{\text {turb }}$ we give the values deduced for the turbulent broadening.

We proved that for the PN with [WC]-type nuclei the internal motions are best represented by a constant expansion velocity with superimposed turbulence. On the contrary the non-[WC] PN show no sign of such high turbulence, they present instead a clear evidence of outward acceleration.

The paper "Turbulent nebulae around [WC]-type stars" (Acker et al. 2002), contains the details of the analysis and the extended discussion.

Table 1. The nebular data and the analysis results

\begin{tabular}{|c|c|c|c|c|c|c|c|c|}
\hline \multirow[t]{2}{*}{ PNG } & \multirow[t]{2}{*}{ name } & \multirow[b]{2}{*}{$\begin{array}{l}\text { c.star } \\
\text { type }\end{array}$} & \multicolumn{3}{|c|}{ planetary nebula } & \multicolumn{3}{|c|}{ results } \\
\hline & & & $\begin{array}{l}\text { diam } \\
\text { arcsec }\end{array}$ & $\begin{array}{l}R_{\text {out }} \\
{[\mathrm{pc}]}\end{array}$ & $\begin{array}{l}M_{\text {ion }} \\
{\left[\mathrm{M}_{\odot}\right]}\end{array}$ & $v_{\exp }$ & $\begin{array}{c}\Delta v \\
{\left[\mathrm{~km} \mathrm{~s}^{-1}\right.}\end{array}$ & $v_{\text {turb }}$ \\
\hline $003.1+02.9$ & $\mathrm{Hb} 4$ & WC & 6.7 & 0.065 & 0.2 & 16 & 0 & 14 \\
\hline 029.2-05.9 & NGC 6751 & WC 4 & 21 & 0.1 & 0.15 & 41 & 0 & 15 \\
\hline $027.6+04.2$ & M 2-43 & WC 8 & 1.6 & 0.02 & 0.04 & 20 & 0 & 10 \\
\hline $327.1-02.2$ & He 2-142 & WC 9 & 3.6 & 0.03 & 0.03 & 20 & 0 & 7 \\
\hline $352.9+11.4$ & K 2-16 & WC 11 & 20 & 0.05 & 0.002 & 34 & 0 & 12 \\
\hline $001.5-06.7$ & SwSt 1 & wels & 1.3 & 0.007 & 0.01 & 17 & 12 & 14 \\
\hline $355.9-04.2$ & M 1-30 & wels & 3.6 & 0.07 & 0.22 & 22 & 28 & 0 \\
\hline $345.2-08.8$ & Tc 1 & $\mathrm{Of}(\mathrm{H})$ & 10 & 0.05 & 0.05 & 20 & 34 & 0 \\
\hline $359.2-33.5$ & CRBB 1 & $\mathrm{O}(\mathrm{H})$ & 9 & 0.08 & 0.18 & 13 & 21 & 0 \\
\hline
\end{tabular}

Acknowledgments. This work was supported by Polish KBN grant No. 2.P03D.020.17 and by French CNRS JUMELAGE programme

\section{References}

Acker et al. 2002, A\&A in press 